\title{
Generalization along the dimension of sound intensity in pigeons*
}

\author{
CHARLES LAWRENCE \\ Departments of Psychiatry \\ Baltimore City Hospitals and The Johns Hopkins University School of Medicine \\ Baltimore, Maryland 21224
}

\begin{abstract}
Two groups of four pigeons each were trained on a discrimination between two intensities of white noise. The low-intensity group had a $60-\mathrm{dB}$ intensity as the negative discriminative stimulus $(\mathrm{S}-)$ and a $70-\mathrm{dB}$ intensity as the positive discriminative stimulus $(S+)$ : the high-intensity group had a $95-\mathrm{dB}$ intensity as $\mathrm{S}-$ and an $85-\mathrm{dB}$ intensity as $\mathrm{S}+$. Generalization stimuli were all of higher intensity than $S+$ for the former group and all of lower intensity than $S+$ for the latter group. The rate of acquisition of the discrimination was faster for the Ss in the high-intensity group. In both groups, the maximum of the generalization function was shifted toward the middle values of the set of test stimuli, away from the training stimuli. Responding showed a decline at the far end of the range of test stimuli. Responding to the positive training stimulus was initially as great as it had been on the preceding training sessions, but became markedly depressed relative to responding to the other stimuli as the test progressed.
\end{abstract}

It has recently been suggested that the form of a stimulus generalization function may depend upon the nature of the stimulus continuum being studied. Heinemann and his associates have found that generalization gradients for white noise intensity following intensity discrimination training do not have the familiar peaked form manifested in generalization tests along such dimensions as line tilt or wavelength of light (Heinemann, Alvin, Sullivail, \& Chase, 1969; Heinemann \& Chase, 1970). They find sigmoidal gradients resembling psychometric functions in both the two-key choice situation and the single-key free operant situation.

There were earlier generalization data for sound intensity which did not show the usual peaked gradients. Pierrel and Sherman (1960) tested rats for generalization along the dimension of tone intensity. They found that Ss trained with the higher of two intensities as the positive discriminative stimulus ( $\mathrm{S}+$ ) showed a marked inflation of responding (i.e., greater than to $S+$ ) to the higher-intensity stimuli presented in the first generalization test. However, Ss trained with the lower-intensity stimulus as $\mathrm{S}+$ showed a decrement in responding to one of the test stimuli lower than $\mathrm{S}+$ and a modest increment in responding to the other lower-than-S+ stimulus. In a later study (Pierrel \& Sherman, 1962), these same investigators presented generalization gradients for rats trained with different

\footnotetext{
*This report is based on a dissertation submitted to the Graduate School, Indiana University, in partial fulfillment of the $\mathrm{PhD}$ degree. The author is grateful to J. A. Dinsmoor for his patience, advice, and encouragement throughout the study. Thanks are also due to Eliot Hearst, whose careful and constructive criticism was most helpful. Reprints may be obtained from the author, Department of Psychiatry, Baltimore City Hospitals, Baltimore, Maryland 21224.
}

amounts of separation between discriminative stimuli (two intensities of a tone). The positive stimulus was always the lower-intensity stimulus, and all of the functions are for generalization to even lower-intensity stimuli. They are very unsystematic, showing increases above St response rate for some new stimuli and decreases for others.

It is interesting that in all of these studies, not only was there a frilure to find the familiar peaked generalization function, but there is an asymmetry to the gradients as well. When S+ was the higher-intensity training stimulus, the Ss always responded maximally (or, in the choice procedure, with the highest probability of being correct) to the most intense generalization stimuli. When $\mathrm{S}+$ was the lower-intensity training stimulus, however, there was not the same inflation of responding (or achievement of the same probability of correct response) to the lowest-intensity stimuli. This asymmetry suggests an influence of absolute stimulus intensity, e.g., a stimulus-intensity dynamism (Hull, 1949).

Thus, it appears that generalization gradients for the dimension of sound intensity may differ in two important ways from those of other stimulus dimensions. First, they are not peaked but are either sigmoidal or irregular; second, they are not symmetrical when comparıng generalization from low to high vs high to low intensities. It is not certain, however, whether these differences are attributable to the nature of the stimulus continuum or to methodological peculiarities of the experiments reviewed. It is possible, for example, that peaked gradients would be found if a wide enough range of stimuli were presented. In an experiment on generalization of light intensity, Ernst, Engberg, and Thomas (1971) consistently found peaked gradients when a wide range of stimuli was presented $(1.5 \mathrm{log}$ 
units beyond $\mathrm{S}+$ ) but not when a smaller range was presented $(0.75 \log$ units). And in another experiment from the same laboratory (Thomas \& Setzer, 1972). peaked gradients were found for generalization of tone intensity with rat and guinea pig Ss. In this study also. a wide range of test stimuli was employed.

The present experiment was designed to provide data relevant to the issue of asymmetry in ascending vs descending generalization functions and to employ as wide a range of test stimuli with pigeons as could reasonably be instrumented.

\section{METHOD}

\section{Subjects}

Eight female White Carneaux pigeons were maintained at $75 \%-80 \%$ of their free-feeding weights. Birds 683. 1745. 1097. 2769 . and 1770 had been used in a previous experiment on autoshaping that included one session of extinction during which the tilt of a black line was varied on a white kev; Bird $1693 \mathrm{had}$ been given one session of continuous reinforcement; Bird 2174 had been given some training on a line-present vs line-absent discrimination; and Bird 1079 had been used in a laboratory demonstration of generalization along the wavelength continuum after training on several schedules of reinforcement. The animals were 6-8 years old at the start of the experiment.

\section{Apparatus}

Two two-key Lehigh Valley Electronics pigeon chambers were used. The right key was transilluminated by white light during stimulus presentations; the other key always remained dark. A $12.7-\mathrm{cm}$ speaker was mounted in the center of a 30.48 x $30.48 \mathrm{~cm}$ section of acoustic tilc and situated against the wall opposite the keys and the hopper. Sections of acoustic tile were also placed on the rear wall and the door. The addition of the tile and the placement of the speaker were designed to minimize variability in the sound level of the stimuli at different locations in the chamber. The ventilating fan was not used.

A Grason-Stadler white noise generator, Model 901B, set at maximum gain provided the sound stimuli. The output of this unit was connected to a set of potentiometers which were set at different values to provide the several degrees of at tenuation of the noise used in training. For generalization tests, the output of the noise generator was connected to a set of L-pad level controls. The intensity levels reported herein were measured with a General Radio sound level meter, Model 1551B. All intensities are in reference to .0002 microbar.

Standard electromechanical relay equipment was used to program the experiment and record the data. This equipment was located in an adjoining room.

Reinforcement consisted of $3-\mathrm{sec}$ access to mixed grain. During reinforcement presentations, the keylight went off and the hopper light came on. The houselight remained on.

\section{Procedure}

\section{Discrimination Training}

All pigeons were retrained to peck the key during their first session, then placed on a $30-\mathrm{sec}$ variable-interval (VI 30-sec) schedule of reinforcement. The noise intensity which was to be the positive discriminative stimulus $(\mathrm{S}+)$ for each animal was continuously present. During the second session, the $\mathrm{S}+$ presentations lasted $30 \mathrm{sec}$ and were separated by 10-5ec blackouts. The keylight. houselight, and noise were all turned of during the blackouts. The negative discriminative stimulus (S- ) was introduced during the third session. One-half of the $30-\mathrm{sec}$ stimulus presentations were of the $S$ - intensity. the other half of the $S+$ intensity On the fourth session. the reinforcement schedule for S+ was changed to $\mathrm{VI} 60 \mathrm{sec}$ and remained at that value for the rest of the experiment. The Ss never received any reinforcement in the presence of $S-$.

The sequence of $S+$ and $S$-. presentations was controlled by 32 steps of a 33-position stepping relay. the order being determined by reference to a table of random digits. The stepping relay cycled five times during each experimental session. providing $80 \mathrm{~min}$ of stimulus presentation, half of which was $S+$ and the other half $S-$. (Birds 1693 and 1770 had several training sessions during which $\mathrm{S}+$ presentations comprised one-third rather than one-half of the stimulus periods.) The Ss were run every day at approximately the same hour until they attained the discrimination criterion. which was that responding in $\mathrm{S}+$ must have comprised at least $90 \%$ of total responding in the session for two consecutive sessions. On the day following the achicvement of criterion, the $S$ received its first generalization test. After that, it was returned to the regular procedure until it met the same criterion and was given a second generalization test.

Birds $683,1745,2174$, and 1693 were trained with a $60-d B$ intensity of noise as $\mathrm{S}$.. and a $70-\mathrm{dB}$ intensity as $\mathrm{S}+$. Birds 1079 1097. 2769. and 1770 were trained with intensities of $95 \mathrm{~dB}$ as $\mathrm{S}-$ and $85 \mathrm{~dB}$ as $\mathrm{S}+$. The ambient noise level in the chamber was $52 \pm 2 \mathrm{~dB}$.

\section{Generalization Tests}

Seven stimulus intensities were presented during the generalization tests: the two training stimuli and five new intensities separated from one another by 5-dB steps. For both groups. the previous $S_{--}$was at one extreme of the test intensities. For the high-intensity discrimination birds, these levels were: $95(\mathrm{~S}-1.85(\mathrm{~S}+) .80 .75,70.65$, and $60 \mathrm{~dB}$. For the low-intensity birds, these levels were $60(\mathrm{~S}-), 70(\mathrm{~S}+), 75,80$. 85. 90 . and $95 \mathrm{~dB}$. Ten blocks of these seven stimuli were presented in the test. The sequence of stimuli within each block was determined by reference to a table of random digits. The test stimuli were changed by the $E$ during the $10 \mathrm{-sec}$ blackont period. The number of responses to each stimulus was recorded on electromechanical counters. The generalization tests were conducted in extinction.

\section{RESULTS}

The group generalization gradients for both tests are presented in Fig. l. Responding is plotted as a function of distance from $\mathrm{S}-$ in order to facilitate comparisons between the two groups. All of the gradients have essentially the same form: a marked peak shift (Hanson, 1959) with maximum responding occurring at an intensity near the middle of the test series and a subsequent decline in responding at the most extreme stimuli. With one exception (1745, first test), all the individual gradients are of the same form. Two examples are shown in Fig. 2. The exceptional gradient showed a response maximum at the highest-intensity stimulus. However, this bird was a very slow responder, and the peak of responding at the highest intensity represents a difference of only some 30 responses above the middle test stimulus. And this bird's second test showed the decline in responding for the most extreme stimuli which characterized all the other gradients. In order to determine whether there was any reliable difference in 


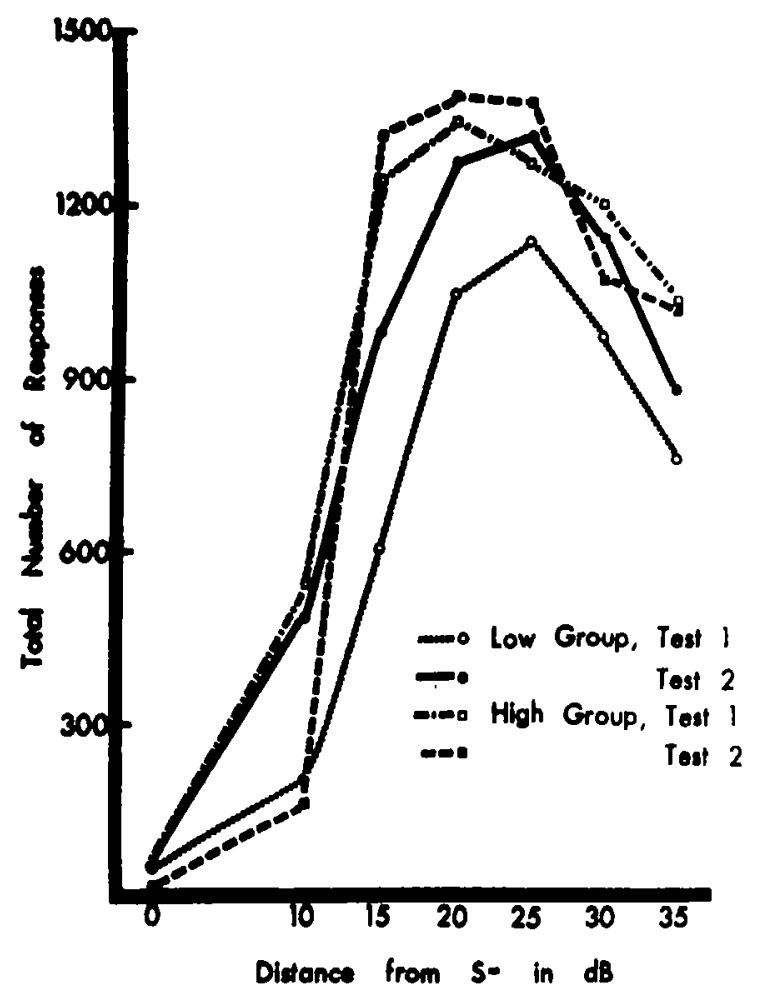

Fig. 1. Generalization gradients for the first and second tests for both groups of Ss. The "10-dB" point represents the previous $S+$ intensity, $70 \mathrm{~dB}$ for the low group and $85 \mathrm{~dB}$ for the high group.

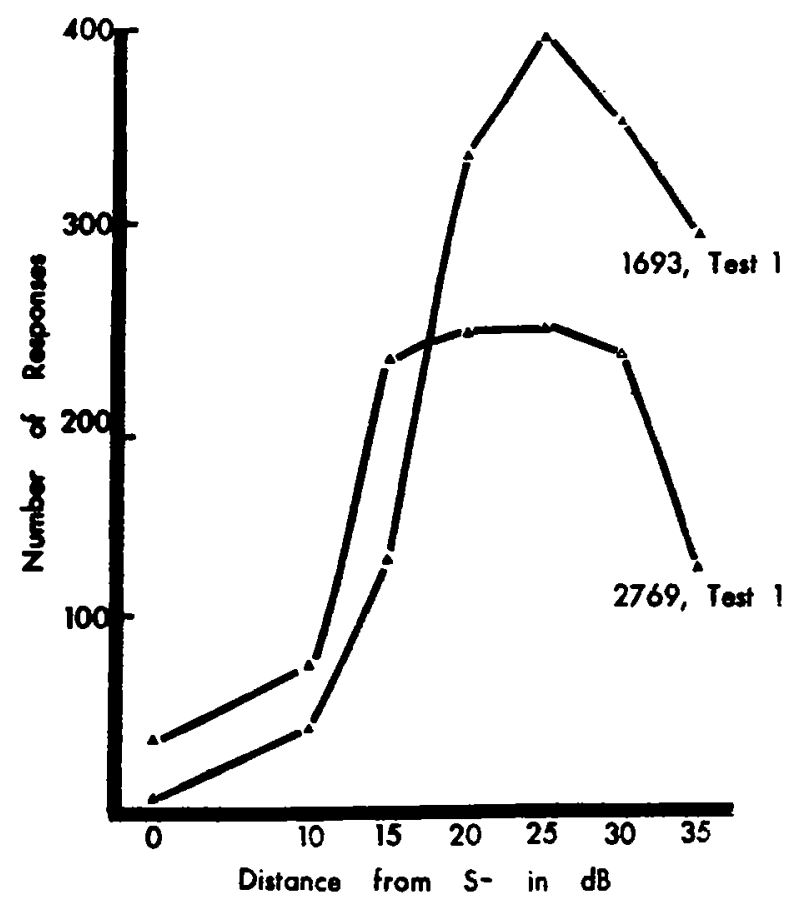

Fig. 2. Individual generalization gradients for a bird trained in the high-intensity condition (2769) and the low-intensity condition (1693).

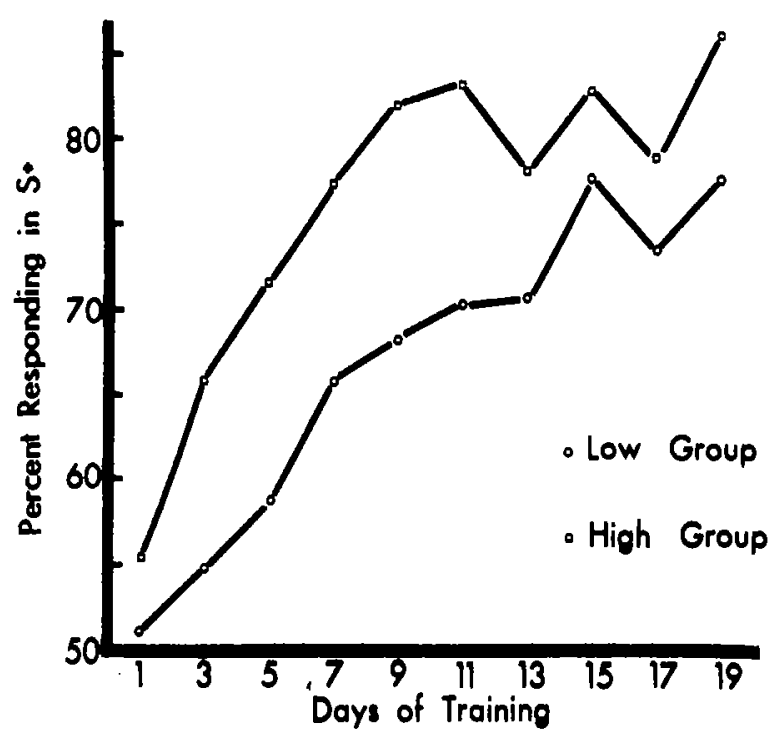

Fig. 3. Graph depicting the rate of acquisition of the discrimination for the group trained with the low-intensity stimuli and the group trained with the high-intensity stimuli.

the form of the gradient between the groups, a 2 by 7 analysis of variance was performed on the data of each test. In this analysis, the A main effect would indicate whether the low-intensity group differed from the high-intensity group over the entire test; the B main effect would indicate whether there is a gradient (which there clearly is); and the $\mathrm{AB}$ interaction would test for proportionality of the two groups at each level of intensity, i.e., whether the functions are parallel. The analyses were carried out on the logarithms of the scores in order to minimize the contribution of overall differences in responding between the groups. For the first test, the $A$ main effect was not significant $(F=$ $3.46, \mathrm{df}=1 / 42)$, the $\mathrm{B}$ main effect was significant $(\mathrm{F}=$ $18.95, \mathrm{df}=6 / 42, \mathrm{p}<.01$ ), and the $\mathrm{AB}$ interaction fell short of significance $(F=0.90, \mathrm{df}=6 / 42)$. Similarly, for the second generalization test, the $\mathrm{A}$ main effect was not significant $(F=0.88, \mathrm{df}=1 / 42)$, the $B$ main effect was highly significant $(F=30.07$, df $=6 / 42, p<.01)$, and the $A B$ interaction was not significant $(F=2.15, \mathrm{df}=$ $6 / 42$ ). One may conclude, therefore, that there were significant differences in each group's responding to the various stimulus intensities presented during the tests, but the null hypothesis of parallel gradients cannot be rejected.

A prominent feature of each of the gradients is the relatively low strength of response to the previous S+. This depression in responding to $\mathrm{S}+$ developed as the generalization test progressed. On the first presentation of S+ during Test $\mathrm{I}$, the rate of response to that stimulus did not differ from the rate on the 2 days preceding the test $(t=.319, .8>p>.5)$, but by the fifth and sixth test presentations, it was significantly lower than on the first presentation $(t=4.57, p<.002)$. The rate of 
responding to the five new stimuli did not drop significantly from the first presentation to the fifth and sixth $(\mathrm{t}=.584, .8>\mathrm{p}>.5)$, so the reduction in $\mathrm{S}+$ responding is not accompanied by an overall reduction in responding during the test. Table 1 presents the individual data on this response depression.

The low-intensity group did not acquire the discrimination as rapidly as the high-intensity group. The respective curves are shown in Fig. 3. All of the points for the Low group are based on the means for the four birds in that group; after Day 10, the means for the High group are based on the three birds which had not yet reached criterion, and after Day 19, only two birds remained in this group, so no further comparisons seemed warranted. A sign test on the mean proportions of $\mathrm{S}+$ responses for the two groups confirms the significance of the superiority of the high-intensity birds in the initial rate of acquisition of the discrimination $(\mathrm{p}<.0001)$.

\section{DISCUSSION}

The results of this experiment indicate that peaked generalization gradients are found along the dimension of sound intensity, just as they are found for other stimulus continua. Furthermore, the gradients were symmetrical for both ascending and descending test intensities. These data are generally compatible with an interacting-gradient account of discrimination learning and generalization, as proposed by Spence (1937).

There is, however, one feature of the present findings that is not derivable from the Spence model: the depression of responding to $\mathrm{S}+$. This was a very reliable phenomenon, evident in the test results for every bird, and suggests the influence of stimulus context. The importance of stimulus context has been illustrated in other studies involving the presentation of several stimulus values: e.g., Garner (1954) presented Ss with one sound intensity as the standard and then gave them several intensities from which to choose a sound half as loud as the standard. Different groups of Ss received different ranges of test stimuli, and it was found that the Ss' judgments of the half-as-loud intensity were determined by the range presented, not by the intensity of the standard. Similarly, Thomas and Jones (1962) found that Ss tended to pick the middle wavelength of a series of test stimuli when requested to identify a previously viewed standard from among them.

Although these two studies used human Ss, the results were similar to those found with pigeons in the present experiment. The birds tended to respond maximally to a stimulus toward the middle of the test series, not to the previous $\mathrm{S}+$. If the animals had been responding on the basis of the relatively higher (or lower) stimulus during training, i.e., not on the basis of absolute intensity, then the introduction of the test stimuli would have altered the reference point (adaptation level), causing a shift in the judgment of the correct intensity. This seems to be
Table 1

Response Rates of Individual Birds to $S+$ and to the Five New Stimuli on the First Presentation in the Generalization Test, and the Average for the Fifth and Sixth Presentations (Upper Four Birds, High Group; Lower Four Birds, Low Group

\begin{tabular}{|c|c|c|c|c|}
\hline & \multicolumn{4}{|c|}{ Responses Per Minute } \\
\hline & \multicolumn{2}{|c|}{ S+ } & \multicolumn{2}{|c|}{$\begin{array}{l}\text { Mean for Five } \\
\text { New Stimuli }\end{array}$} \\
\hline & First & $\begin{array}{l}\text { Fifth } \\
\text { and } \\
\text { Sixth }\end{array}$ & First & $\begin{array}{c}\text { Fifth } \\
\text { and } \\
\text { Sixth }\end{array}$ \\
\hline 2769 & 62.0 & 0.0 & 42.0 & 35.2 \\
\hline 1079 & 42.0 & 3.0 & 65.6 & 59.6 \\
\hline 1097 & 74.0 & 16.0 & 72.8 & 65.6 \\
\hline 1770 & 78.0 & 37.0 & 100.0 & 76.8 \\
\hline 2174 & 34.0 & 3.0 & 38.0 & 37.6 \\
\hline 1745 & 14.0 & 2.0 & 16.0 & 12.4 \\
\hline 683 & 108.0 & 3.0 & 70.0 & 70.8 \\
\hline 1693 & 64.0 & 0.0 & 88.4 & 74.8 \\
\hline
\end{tabular}

the most reasonable way to account for the location of the point of maximum responding toward the middle of the test series, and it could also account for the reduction of responding to $\mathrm{S}+$ that developed during the test. The pigeon $\mathrm{Ss}$ in this experiment behaved much like the human Ss in the experiments reviewed above. They identified a stimulus toward the middle of the test series as the "standard" (previous $S+$ ). The reduction in responding to St during the test could have occurred because this stimulus was related to the context-determined "S+" (the middle test stimulus) in the same way as the original $S$ was related to the original S+, i.e., relatively higher or lower in intensity.

The location of the point of maximum responding also represents a peak shift (Hanson, 1959); however. peak shift has been shown by Terrace (1966) to disappear after extended discrimination training (60 sessions). Four of the birds in the present experiment took over 40 sessions to meet criterion for the first time. and one took over 71. Yet all the Ss show the same shift of maximum responding toward the middle of the test series. Apparently, extended training has no effect on the peak shift in postdiscrimination gradients for auditory intensity, or else the mechanism responsible for the shift is not the same for this dimension as for others such as wavelength of light or angle of line tilt.

The fact that the gradients for ascending and descending intensities were symmetrical deserves further comment. In the earlier studies of generalization of sound intensity (Pierrel \& Sherman, 1960, 1962; Heinemann et al, 1969; Heinemann \& Chase, 1970), the gradients for ascending intensities showed a regular and pronounced inflation of rate (or an increase in proportion of correct responses), whereas the gradients for descending intensities were irregular in form. Such results offered some support for Hull's notion of a stimulus-intensity dynamism (Hull, 1949). The symmetrical gradients found here provide no support for that notion. 
It is difficult to find a particular factor which accounts for the differences between the present results and past findings. In respect to the form of the gradients, the range of test stimuli may have been important. Most of the earlier studies on generalization of sound intensity employed a narrower range of test stimuli than that used here or a similar range but spread on both sides of the S+ intensity. Also, in all but one of the experiments cited above, reinforcement to $\mathrm{S}+$ was maintained during testing. In the one case where an extinction test was used (Heinemann \& Chase, 1970, Experiment II), the data presented do not distinguish between gradients for ascending vs descending intensities, so the presence or absence of symmetry cannot be determined. In any event, it is not intuitively clear why the maintenance of reinforcement should differentially affect generalization for ascending and descending intensities. Perhaps some other factor which is not immediately evident was responsible.

Although absolute stimulus intensity did not produce differential effects on the generalization gradients, it did exert an influence on the Ss' performance during training. The birds trained with the more intense stimulus pair acquired the discrimination at a more rapid rate. This finding corroborates those reported by Pierrel et al (1970) and by Thomas and Setzer (1972). In both of these studies, the discrimination was learned faster when the stimuli were of higher intensity than when they were of lower intensity. The more intense stimuli could be more "attention-demanding."

In conclusion, it can be stated with certainty that when a wide range of stimuli are presented in a generalization test carried out during extinction, the postdiscrimination gradients for sound intensity show the form characteristic of generalization functions in other stimulus dimensions. The gradients exhibit a pronounced peak shift, but whether this supports the Spence (1937) model of discrimination learning is equivocal. That model would not predict the reduction in $S+$ responding found here, while a stimulus-context or adaptation-level explanation could account for both this reduction and the location of maximum responding.

\section{REFERENCES}

Ernst, A. J., Engberg, L., \& Thomas, D. R. On the form of stimulus generalization curves for visual intensity. Journal of the Experimental Analysis of Behavior, 1971, 16, 177-180.

Garner, W. R. Context effects and the validity of loudness scales. Journal of Experimental Psychology, 1954, 48, 218-224.

Hanson, H. M. Effects of discrimination training on stimulus generalization. Journal of Experimental Psychology, 1959, 58, 321-334.

Heinemann, E. G., Alvin, E., Sullivan, M., \& Chase, S. Analysis of stimulus generalization with a psychophysical method. Journal of Experimental Psychology, 1969, 80, 215-224.

Heinemann, E. G., \& Chase, S. On the form of stimulus generalization curves for auditory intensity. Journal of Experimental Psychology, 1970, 84, 483-486.

Hull, C. L. Stimulus-intensity dynamism (V) and stimulus generalization. Psychological Review, 1949, 56, 67-76.

Pierrel, R., \& Sherman, J. G. Generalization of auditory intensity following discrimination training. Journal of the Experimental Analysis of Behavior, 1960, 3, 313-322.

Pierrel, R., \& Sherman, J.G. Generalization and discrimination as a function of $S^{D}-S$ intensity difference. Journal of the Experimental Analysis of Behavior, 1962, 5, 67-71.

Pierrel, R., Sherman, J. G., Blue, S., \& Hegge, F. W. Auditory discrimination: A three variable analysis of intensity effects. Journal of the Experimental Analysis of Behavior, 1970, 13, 17-35.

Spence, K. W. The differential response in animals to stimuli varying within a single dimension. Psychological Review, $1937,44,430-444$.

Terrace, H. S. Behavioral contrast and the peak shift: Effects of extended discrimination training. Journal of the Experimental A nalysis of Behavior, 1966, 9, 613-617.

Thomas, D. R., \& Jones, C. G. Stimulus generalization as a function of frame of reference. Journal of Experimental Psychology, 1962, 64, 81-86.

Thomas, D., \& Setzer, J. Stimulus generalization gradients for auditory intensity in rats and guinea pigs. Psychonomic Science, 1972, 28, 22-24.

(Received for publication July 31,1972 ; revision received October 20,1972.) 